\title{
Comportamento de citrumelos em área afetada pela morte súbita dos citros
}

\author{
Performance of citrumelos in area affected by citrus sudden death \\ Comportamiento de citrumelos en zona afectada por la muerte súbita de los cítricos
}

Recebido: 08/12/2021 | Revisado: 17/12/2021 | Aceito: 28/12/2021 | Publicado: 06/01/2022

\author{
Jorgino Pompeu Junior \\ ORCID: https://orcid.org/0000-0002-2189-6411 \\ Instituto Agronômico do Estado de São Paulo, Brasil \\ E-mail: jorginopompeu@gmail.com \\ Silvia Blumer \\ ORCID: https://orcid.org/0000-0002-6893-7639 \\ Centro Universitário Fundação de Ensino Octavio Bastos, Brasil \\ E-mail: silvia.blumer@unifeob.pro.br \\ Josiane Rodrigues \\ ORCID: https://orcid.org/0000-0003-0365-6227 \\ Universidade Federal de São Carlos, Brasil \\ josirodrigues@ufscar.br \\ Eduardo Sanches Stuchi \\ ORCID: https://orcid.org/0000-0001-6194-0117 \\ Empresa Brasileira de Pesquisa Agropecuária: Mandioca e Fruticultura, Brasil \\ E-mail: stuchi@embrapa.br \\ Eduardo Augusto Girardi \\ ORCID: https://orcid.org/0000-0003-4839-5227 \\ Empresa Brasileira de Pesquisa Agropecuária: Mandioca e Fruticultura, Brasil \\ E-mail: eduardo.girardi@embrapa.br
}

\begin{abstract}
Resumo
A morte súbita dos citros (MSC) é uma doença biótica de origem desconhecida, transmitida por vetores aéreos e borbulhas, que afeta cultivares de laranjas doces e tangerinas enxertadas nos limoeiros Cravo, Volkameriano e Rugoso. As mesmas cultivares enxertadas nas tangerinas Cleópatra e Sunki, trifoliata e citrumelo Swingle são assintomáticas. O objetivo deste trabalho foi avaliar nove seleções de citrumelo quanto à tolerância à MSC e seus efeitos no desenvolvimento de laranjeiras Valência cultivadas sem irrigação. Utilizou-se o delineamento inteiramente casualizado com nove tratamentos (citrumelos) e cinco repetições sendo cada parcela representada por uma planta. Após 16 anos no campo, nenhuma planta apresentou sintomas de MSC ou anel de goma (bud union ring) entre a copa e o porta-enxerto. Dados de 14 colheitas controladas revelam que as laranjeiras Valência enxertadas nos citrumelos Swingle, W-2, F80-7 e F80-8 apresentaram as maiores produções sendo que W-2 e F80-7 induziram produção precoce e não motivaram alternância de produção. Os citrumelos W-2, Swingle e F80-7 formaram as plantas mais altas e os citrumelos F80-5, F80-3 e F81-18 induziram as árvores mais baixas. Embora não proporcionarem elevada produção de frutos por planta, estes três porta-enxertos apresentaram maior eficiência produtiva, e o F80-5 motivou também produção precoce e menor diâmetro de copa, possibilitando maior densidade de plantio.
\end{abstract}

Palavras-chave: Citrus spp.; Poncirus trifoliata; Doença; Porta-enxerto; Híbrido de trifoliata.

\begin{abstract}
Citrus sudden death (CSD) is a biotic disease of unknown origin, transmitted by aerial vectors and buds that affects sweet oranges and tangerines cultivars budded on Rangpur lime, and Volkamer and Rough lemons rootstocks. The same cultivars onto Cleopatra and Sunki mandarins, trifoliate orange and Swingle citrumelo are asymptomatic. The objective of this study was to evaluate nine selections of citrumelo regarding tolerance to CSD and their effects on growth and production of Valencia sweet orange trees cultivated without irrigation. A completely randomized design with nine treatments (citrumelos) and five replicates, each plot represented by one tree. After 16 years in the field, no tree showed symptoms of CSD or bud union ring between the scion and the rootstock. After 14 yields, the trees onto Swingle, W-2, F80-7 and F80-8 yielded the highest productions and W-2 and F80-7 induced yearly production and did not show alternate bearing. The W-2, Swingle and F80-7 citrumelos induced the largest trees and the F80-5, F80-3 and F81-18 the smaller trees. Although they do not induce high fruit production per tree, they have the highest production efficiencies, and F80-5 motivated early production and a smaller canopy diameter, enabling greater planting density.
\end{abstract}

Keywords: Citrus spp.; Poncirus trifoliata; Disease; Rootstock; Trifoliate orange hybrid. 


\begin{abstract}
Resumen
La muerte súbita de los cítricos (MSC) es una enfermedad biótica de origen desconocida, transmitida por vectores aéreos y por yemas, que afecta a cultivares de naranjas dulces y mandarinas injertados en los limoneros Rangpur, Volkameriano y Rugoso. Los mismos cultivares injertados en las mandarinas Cleopatra y Sunki, trifoliados y citrumelo Swingle son asintomáticos. El objetivo de este trabajo fue evaluar nueve selecciones de citrumelo respecto a la tolerancia a MSC y sus efectos sobre el crecimiento y producción de naranjeiras Valencia crecido sin riego. Se utilizó un diseño completamente aleatorizado con nueve tratamientos (citrumelos) y cinco réplicas, cada qual representada por una planta. Después de 16 años en el campo, ningún árbol mostró síntomas de MSC o anillo de goma entre el dosel y el portainjerto. Los datos de 14 cocechas muestram que los citrumelos Swingle, W-2, F80-7 y F80-8 produjeron las mayores producciones y W-2 y F80-7 indujeron producción temprana y no mostraron alternancia de producción. Las plantas más altas estaban en los citrumelos W-2, Swingle y F80-7 e las mas bajas em los citrumelos F80-5, F80-3 y F81-18. Aunque no inducen alta producción de fruta por planta, estos citrumelos tienen las mayores eficiencias de producción y el F80-5 también motivó producción temprana y un diámetro de dosel más pequeño, lo que permite una mayor densidad de plantio.
\end{abstract}

Palabras clave: Citrus spp.; Poncirus trifoliata; Enfermedad; Portainjerto; Híbrido trifoliado.

\title{
1. Introdução
}

A morte súbita dos citros (MSC), constatada no sul do Triângulo Mineiro em 1999, vem causando o definhamento e morte de laranjeiras doces (Citrus sinensis (L.) Osbeck) e tangerineiras Cravo e Ponkan (C. reticulata Blanco) com dois ou mais anos de idade enxertadas nos limoeiros Cravo (C. limonia L. Osbeck), Volkameriano (C. volkameriana V. Tennore et Pasquale) e Rugoso (C. jambhiri Lushington) (Bassanezi et al., 2007, 2016). Ela prossegue expandindo-se por pomares do Triângulo Mineiro e do Norte e do Noroeste do estado de São Paulo, não tendo sido constatada em outras regiões citrícolas brasileiras.

As folhas das plantas afetadas perdem o brilho, adquirem coloração verde-pálido, seguindo-se a queda das folhas e morte dos ramos apicais, decorrentes da redução do sistema radicular em consequência da degeneração dos tecidos do floema abaixo da região da enxertia, culminando com a morte das plantas, em poucas semanas a dois anos após o aparecimento dos sintomas. A retirada da casca dos porta-enxertos das plantas comprometidas revela o amarelecimento na região cambial, sendo esse o sintoma-diagnóstico da doença e que, geralmente, precede os sintomas da copa (Bassanezi et al., 2016).

Plantas enxertadas nas tangerinas Cleópatra (C. reshni hort. ex Tanaka) e Sunki [C. sunki (Hayata) hort. ex Tannaka], trifoliata (Poncirus trifoliata (L.) Rafinesque) e citrumelo Swingle [C. paradisi Macfaden $\times P$. trifoliata (L.) Rafinesque] são assintomáticas, sendo estes porta-enxertos considerados tolerantes à MSC. Entretanto, borbulhas retiradas dessas plantas são capazes de induzir sintomas da doença em combinações sadias indicando que a MSC é causada por agente biótico e pode estar presente nas plantas enxertadas e nas subenxertadas com porta-enxertos tolerantes (Yamamoto et al., 2011).

Presume-se que seja causada por variantes do vírus da tristeza do citros (CTV) e/ou pelo CSDaV (Citrus Sudden Death associated virus) associados a outros vírus e transmitida por vetores aéreos e por borbulhas (Roman et al., 2004; Maccheroni et al., 2005; Yamamoto et al., 2011; Bassanezi et al., 2016; Matsumura et al., 2017).

A convivência com a MSC tem sido feita utilizando os porta-enxertos considerados tolerantes, tangerinas Cleópatra e Sunki, trifoliatas e citrumelo Swingle, todos mais suscetíveis a seca do que o limão Cravo, fragilizando ainda mais a citricultura.

A inserção de inter-enxerto de trifoliata ou de tangerina Cleópatra entre o limoeiro Cravo e a laranjeira Valência $(C$. sinensis (L.) Osbeck) visando "filtrar" o agente causal e preservar a utilização desse porta-enxerto, essencial para uma citricultura não irrigada, não impediu a manifestação da doença (Pompeu Junior \& Blumer, 2008a).

Visando ampliar a diversificação de porta-enxertos tolerantes à MSC, mais de duas centenas de variedades e espécies cítricas estão sendo avaliadas em áreas sujeitas ao problema desde 2003 (Pompeu Junior \& Blumer, 2008a; Blumer \& Pompeu Junior, 2010; Pompeu Junior et al., 2013; Pompeu Junior \& Blumer, 2019; Costa et al., 2020; 2021). 
A suscetibilidade do limão Cravo ao declínio e à morte súbita dos citros (Rodriguez et al., 1979; Bassanezi et al., 2003) e a tolerância do citrumelo Swingle a essas doenças (Beretta et al.,1994; Roman et al., 2004) acrescida da sua grande resistência à gomose de Phythophtora nicotianae e melhor qualidade dos frutos sobre ele produzidos tornaram-no, a partir de 2001, o segundo porta-enxerto mais utilizado pelos citricultores paulistas (Pompeu Junior \& Blumer, 2008b); em 2019, o citrumelo Swingle estava presente em 50\% das mudas dos viveiros comerciais (Fundecitrus, 2021).

Originado pelo cruzamento do pomelo Duncan (C. paradisi Macfaden) com P. trifoliata Rafinesque realizado por Walter T. Swingle em 1907, na Florida, EUA, com objetivo de transferir a resistência ao frio dos trifoliatas para o pomelo e inicialmente denominado de CBP 4475, o citrumelo Swingle é tolerante à tristeza, à exocorte, à xiloporose, ao declínio e à morte súbita dos citros, e resistente à gomose por Phytophtora e ao nematoide dos citros (Tylenchulus semipetrans), porém, é mais intolerante à seca que o limão Cravo e incompatível com diversos clones da laranja Pera (C. sinensis (L.) Osbeck) e o tangor Murcott (C. sinensis (L.) Osbeck $\times$ C. reticulata Blanco), exigindo a utilização de inter-enxerto, compatível com as duas partes envolvidas, para a formação de plantas longevas e produtivas (Pompeu Junior, 2005; Pompeu Junior \& Blumer, 2020).

Em 1955, o Dr. Mortimer Cohen, da Universidade da Flórida, repetiu o cruzamento do pomelo Duncan com trifoliata, dando origem a citrumelos genericamente designados de F80 e F81 (Wutscher et al.,1988), parte dos quais foram introduzidos pelo Centro de Citricultura Sylvio Moreira do Instituto Agronômico de Campinas em 1990 e 1993.

Tendo em vista as boas características do citrumelo Swingle e a necessidade de ampliar o número de porta-enxertos tolerantes à MSC, este estudo foi conduzido com o objetivo de avaliar o comportamento de seleções de citrumelo na presença dessa doença e suas influências no desenvolvimento da laranjeira (C. sinensis (L.) Osbeck) cv. Valência.

\section{Material e Métodos}

Utilizando mudas produzidas a partir de sementes coletadas no Banco Ativo de Germoplasma de Citros do Instituto Agronômico de Campinas, foram avaliadas nove seleções de citrumelo: Swingle (4475), 4481, W-2, F80-3, F80-5, F80-6, F807, F80-8, F80-14, F80-18 e F81-18.

Como copa, foi selecionada a laranjeira Valência (C. sinensis (L.) Osbeck) IAC, clone nucelar, inoculado com o isolado protetivo Pera IAC (PIAC) contra a virose tristeza (Muller et al., 1999). A eleição dessa variedade deveu-se ser ela a segunda cultivar-copa mais importante da citricultura paulista, ser suscetível à MSC quando enxertada nos limoeiros Cravo e Volkameriano (Bassanezi et al., 2003) e compatível com a quase totalidade dos porta-enxertos, exceto o limoeiro Rugoso (Bridges \& Youtsey, 1968) e o hibrido limão Cravo x citrange Carrizo [C. limonia x (P. trifoliata x C. sinensis)] (Pompeu Júnior \& Blumer, 2002). A utilização da laranjeira Pêra (C. sinensis), resultaria na incompatibilidade com os citrumelos Swingle, F80-3 e F80-5 (Pompeu Junior \& Blumer, 2005), o que prejudicaria a interpretação dos resultados.

O experimento foi instalado em maio de 2003, em Comendador Gomes, MG, coordenadas 19²41'54" Sul e 49 $04^{\prime} 50^{\prime \prime}$ Oeste, 557 m de altitude (www.geografos.com.br), clima tropical Aw (Alvares et al., 2013) em Latossolo Vermelho-Amarelo Distrófico Psamitico (Embrapa, 1999), no espaçamento 7,5 m x 3,5 m e conduzido sem irrigação, em área anteriormente ocupada por pomares erradicados pela presença da MSC e próxima a pomares afetados por ela, parte dos quais havia sido subenxertado com porta-enxertos tolerantes perpetuando a doença (Yamamoto et al., 2011).

À semelhança de outros estudos que avaliaram a reação de elevado número de genótipos de citros a infecções por fito patógenos (Moreira et al., 1965; Laranjeira et al., 1998), foram plantadas conjuntos de cinco mudas de cada combinação, agrupadas pela espécie ou pelos parentais (ex. limões-cravos, citrumelos etc.), visando a facilitar as observações e comparações entre os porta-enxertos, em sua maioria inéditos no Brasil, que mostrassem tolerância à MSC. Foram também 
plantados conjuntos de cinco mudas de laranja Valência enxertadas em limão Cravo, distribuídas aleatoriamente pelo pomar experimental (Pimentel Gomes, 2009) destinadas a indicar a presença da doença no experimento.

Os tratamentos foram comparados pela altura e diâmetro das copas das plantas em outubro de 2009, pela produção individual anual e total de frutos por planta no quinquênio 2006-2010, precocidade e alternância na produção dos frutos e pela eficiência produtiva das copas.

A precocidade da produção (PP) foi estimada pela percentagem da produção obtida nas três primeiras colheitas em relação ao total de frutos colhidos nas cinco safras controladas (Cantuaria-Aviles et al., 2011).

O índice de alternância da produção dos frutos (IAP) foi calculado de 2006 a 2010, utilizando-se a expressão IAP = 1/ $(n-1) *\left[\left(a_{2}-a_{1}\right) /\left(a_{2}+a_{1}\right)+\left(a_{3}-a_{2}\right) /\left(a_{3}+a_{2}\right)+\ldots+\left(n_{a}-a_{n-1}\right) /\left(n_{a}+a_{n-1}\right)\right]$, em que ' $n$ ' representa o número de anos e ' $a_{1}, a_{2}$, $\ldots, \mathrm{a}_{\mathrm{n}-1}, \mathrm{a}_{\mathrm{n}}$ ' a produção de frutos, nos anos correspondentes. O IAP pode variar de zero a um, e quanto mais próximo da unidade, maior é a alternância da produção (Stenzel et al., 2005).

A eficiência produtiva (EP) foi calculada pelo quociente da produção média de frutos no biênio 2009-2010, para compensar a alternância de produção, pelo volume médio das copas em outubro de 2009 , utilizando a formula: $V=2 / 3 \pi R^{2} H$, onde R é o raio médio das copas e H a altura das plantas (Mendel, 1956).

Os dados obtidos foram submetidos a análises estatísticas utilizando o software R Core Team (2020) e as médias comparadas pelo Teste de Tukey a 5\% de probabilidade (Tukey, 1949).

As plantas foram avaliadas a cada três a quatro meses, sendo uma delas antecedendo a colheita, quanto à presença de sintomas da MSC e incompatibilidade entre a cultivar Valência e os porta-enxertos.

Em 2008, foram excluídos os tratamentos F80-18 e F80-14 por apresentarem crescimento desuniforme das copas, sugerindo baixa embrionia nucelar, e baixíssimas produções de frutos nas colheitas de 2006 e 2007.

\section{Resultados e Discussão}

Em dezembro de 2006, sintomas da MSC estavam presentes em laranjeiras Valência sobre diversas seleções de limão Cravo, próximas deste experimento, indicando a presença da doença na área experimental (Pompeu Junior \& Blumer, 2008). Até janeiro de 2011, quando as plantas tinham sete anos de idade no pomar experimental, nenhuma delas apresentava sintomas da MSC ou a presença de anel de goma na linha de enxertia.

Na Tabela 1, são apresentadas as produções médias anuais e total de 2006 a 2010 das laranjeiras Valência enxertadas nos nove citrumelos assim como índices de precocidade da produção (PP), dimensões das plantas e eficiência produtiva das copas (EP). 
Tabela 1. Produção de frutos, índice de precocidade, dimensões das plantas e eficiência produtiva de laranjeiras Valência enxertadas em citrumelos. Comendador Gomes - MG.

\begin{tabular}{|c|c|c|c|c|c|c|c|c|c|c|}
\hline \multirow[t]{3}{*}{ Citrumelo } & \multicolumn{6}{|c|}{ Produção } & $\mathrm{PP}^{*}$ & \multirow[t]{3}{*}{ Alt.** } & \multirow{3}{*}{$\begin{array}{l}\text { Diâm.** } \\
\text { m------- }\end{array}$} & \multirow{3}{*}{$\begin{array}{l}\mathrm{EP} * * * \\
\mathrm{~kg} \cdot \mathrm{m}^{-3}\end{array}$} \\
\hline & 2006 & 2007 & 2008 & 2009 & 2010 & $2006-10$ & \multirow[b]{2}{*}{$\%$} & & & \\
\hline & \multicolumn{6}{|c|}{ 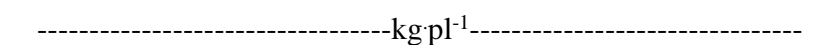 } & & & & \\
\hline Swingle & $23,2 a b c^{1}$ & $55,5 \mathrm{ab}$ & $39,6 \mathrm{ab}$ & $88,0 \mathrm{a}$ & 126,4 a & $332,7 \mathrm{ab}$ & 36 & $2,7 \mathrm{ab}$ & $3,2 \mathrm{ab}$ & 12,5 \\
\hline $\mathrm{W}-2$ & $32,4 \mathrm{a}$ & $64,0 \mathrm{a}$ & 56,0 a & $63,8 \mathrm{ab}$ & $136,4 \mathrm{a}$ & 352,6 a & 43 & $2,9 \mathrm{a}$ & $3,3 \mathrm{a}$ & 13,1 \\
\hline F80-7 & $24,3 \mathrm{ab}$ & $41,8 \mathrm{abc}$ & $46,0 \mathrm{ab}$ & $54,1 \mathrm{ab}$ & $109,4 a b c$ & $275,6 \mathrm{abc}$ & 41 & $2,6 a b$ & $2,9 \mathrm{abc}$ & 11,9 \\
\hline F80-8 & $17,8 \mathrm{bc}$ & $37,7 \mathrm{bcd}$ & $31,4 \mathrm{ab}$ & $56,6 \mathrm{ab}$ & $110,0 \mathrm{abc}$ & $253,5 \mathrm{a}-\mathrm{d}$ & 34 & $2,4 \mathrm{bc}$ & 2,7 a-d & 13,19 \\
\hline F80-5 & $14,5 \mathrm{bc}$ & $33,3 \mathrm{bcd}$ & $49,0 \mathrm{ab}$ & $34,5 \mathrm{~b}$ & $94,6 \mathrm{abc}$ & $225,9 \mathrm{bcd}$ & 43 & $1,9 \mathrm{~cd}$ & $2,6 \mathrm{bcd}$ & 15,3 \\
\hline F81-18 & $12,3 \mathrm{bc}$ & $16,4 \mathrm{~d}$ & $38,4 \mathrm{ab}$ & $35,2 \mathrm{~b}$ & $90,2 \mathrm{abc}$ & $192,6 \mathrm{~cd}$ & 35 & $2,0 \mathrm{~cd}$ & $2,3 \mathrm{~cd}$ & 15,7 \\
\hline F80-6 & $10,7 \mathrm{bc}$ & $30,0 \mathrm{~cd}$ & $32,1 \mathrm{ab}$ & $40,4 \mathrm{~b}$ & $78,8 \mathrm{bc}$ & $191,9 \mathrm{~cd}$ & 38 & $2,3 \mathrm{bc}$ & $2,5 \mathrm{bcd}$ & 11,2 \\
\hline 4481 & $9,4 \mathrm{c}$ & $23,3 \mathrm{~cd}$ & $19,6 \mathrm{~b}$ & $44,0 \mathrm{~b}$ & $72,8 \mathrm{bc}$ & $169,1 \mathrm{~cd}$ & 31 & $2,0 \mathrm{~cd}$ & $2,3 \mathrm{~cd}$ & 12,4 \\
\hline F80-3 & $14,8 \mathrm{bc}$ & $24,2 \mathrm{~cd}$ & $25,6 \mathrm{ab}$ & $37,6 \mathrm{~b}$ & $55,0 \mathrm{c}$ & $157,2 \mathrm{~d}$ & 41 & $1,7 \mathrm{~d}$ & $2,1 \mathrm{~d}$ & 14,6 \\
\hline C. V. $\%$ & 38,1 & 32,7 & 42,5 & 38,2 & 27,4 & 21,9 & $\cdots$ & 9,7 & 12,6 & 24,6 \\
\hline
\end{tabular}

Neste período, o citrumelo W-2 induziu a maior produção $\left(352,6 \mathrm{~kg}^{\mathrm{p}} \mathrm{pl}^{-1}\right)$ sem diferir estatisticamente dos citrumelos Swingle (332,7 kg pl-1), F80-7(275,6 kg pl-1) e F80-8 (253,5 $\left.\mathrm{kg}^{-1} \mathrm{pl}^{-1}\right)$, sendo que W-2 e F80-7 apresentaram PP (43\%) e (41\%) ambos maiores que os do Swingle (36\%) e do F80-8 (34\%). Com comportamento mais tardio, o citrumelo F80-8 somente apresentou bom desempenho nas três últimas colheitas desse período, igualando-se então aos três porta-enxertos mais produtivos no conjunto das cinco colheitas.

Os citrumelos F81-18, F80-6, 4481 e F80-3 motivaram as menores produções totais: 192,6; 191,9; 169,1 e 157,2 kg $\mathrm{pl}^{-}$ ${ }^{1}$, respectivamente, todas inferiores à média geral do período, $239,0 \mathrm{~kg} \cdot \mathrm{pl}^{-1}$.

Os dados de altura e diâmetro das copas (Tabela 1), coletados em outubro de 2009 quando o experimento tinha sete anos de idade, mostram que os citrumelos W-2, Swingle e F80-7, sem diferirem estatisticamente entre si, proporcionaram as plantas mais altas $(2,9 ; 2,7$ e 2,6 m) e com maior diâmetro de copa $(3,3 ; 3,2$ e 2,9 m), respectivamente, enquanto que os demais deram formação a plantas menores. Sem diferirem entre si, os citrumelos F80-3 e F80-5 desenvolveram as plantas de menor altura $(1,7$ e $1,9 \mathrm{~m})$ e diâmetro da copa $(2,1 \mathrm{~m}$ e 2,6 m) do experimento.

Sem diferirem estatisticamente, os citrumelos W-2, Swingle, F80-8 e F80-7 apresentaram as menores EP: 13,1; 12,6; 13,0 e 12,0 $\mathrm{kg} \cdot \mathrm{m}^{-3}$, respectivamente, sendo as maiores induzidas pelos F81-18 (15,7 kg $\left.\mathrm{m}^{-3}\right)$ e $\mathrm{F} 80-5\left(15,4 \mathrm{~kg} \cdot \mathrm{m}^{-3}\right)$.

Apesar de não ter sido constatada diferença estatística entre os irmãos F80-3 e F80-5, este produziu, neste período, $31 \%$ mais frutos do que o F80-3 (225,9 contra 157,2 $\left.\mathrm{kg} \cdot \mathrm{pl}^{-1}\right)$, apresentou maior PP $(43 \% ; 41 \%)$ e EP $\left(15,4 \mathrm{~kg} \cdot \mathrm{m}^{-3} ; 14,6 \mathrm{~kg} \cdot \mathrm{m}^{-3}\right)$, características que o tornam interessante para plantios adensados.

Os dados de alternância de produção, que variaram de 0,01 a 0,18 não mostraram diferenças significativas entre os citrumelos (Tukey $5 \%$ de probabilidade).

Em outubro de 2009, ao final do período de seca na região, o enrolamento foliar, característico de planta sob estresse hídrico, mostrava que todas as combinações apresentavam boa tolerância à seca, semelhante à das laranjeiras enxertadas nos limões cravos EEL, Limeira e Taquaritinga, combinações até então assintomáticas para MSC.

A partir de 2011, os frutos foram coletados abarcando as cinco plantas de cada tratamento, o que impossibilitou a análise estatística dos dados de produção. Na Tabela 2, são apresentadas as produções médias anuais e total de frutos de 2011 a 
2019 dos tratamentos, as dimensões das plantas em outubro de 2019 e a eficiência produtiva das copas calculada para o biênio 2018 e 2019.

Tabela 2. Produção de frutos, dimensões das plantas e eficiência produtiva de laranjeiras Valência enxertadas em citrumelos. Comendador Gomes - MG.

\begin{tabular}{|c|c|c|c|c|c|c|c|c|c|c|c|c|c|c|}
\hline \multirow[t]{2}{*}{ Porta - enxerto } & \multicolumn{11}{|c|}{ Produção } & Alt* & Diâm* & $\mathrm{EP} * *$ \\
\hline & 2011 & 2012 & 2013 & 2014 & 2015 & 2016 & 2017 & 2018 & 2019 & 2011-19 & Total $* * *$ & \multirow{2}{*}{\multicolumn{2}{|c|}{----m---- }} & \multirow[b]{2}{*}{$\mathrm{kg} \cdot \mathrm{m}^{-3}$} \\
\hline & & & & & & & $\mathrm{kg} \cdot \mathrm{pl}^{-1}$ & & & & --- & & & \\
\hline Citrumelo Swingle & 93,6 & 113 & 137,2 & 160 & 115,8 & 83,8 & 249,2 & 87,2 & 151,6 & 1191,5 & 1524,2 & 4,1 & 4,6 & 2,6 \\
\hline Citrumelo 4481 & 49,7 & 72,8 & 59,4 & 101,6 & 68,8 & 18,2 & 137 & 59,7 & 65,0 & 632,2 & 801,2 & 3,3 & 3,7 & 2,5 \\
\hline Citrumelo W-2 & 105,9 & 122,6 & 99,6 & 158,2 & 105 & 95,8 & 290,7 & 110,6 & 105,4 & 1193,8 & 1546,4 & 4,2 & 5,3 & 1,7 \\
\hline Citrumelo F80-6 & 80,1 & 80,6 & 44,4 & 132,6 & 77,8 & 58,5 & 157,5 & 157,1 & 37,0 & 825,7 & 1017,5 & 3,6 & 4,9 & 2,1 \\
\hline Citrumelo F80-5 & 67,1 & 61,6 & 21,8 & 114,4 & 76,8 & 17,7 & 135,5 & 36,9 & 73,8 & 605,5 & 831,5 & 2,9 & 3,8 & 2,6 \\
\hline Citrumelo F80-7 & 125,5 & 99,0 & 84,0 & 84,2 & 74,2 & 62,0 & 271,8 & 100,7 & 166,0 & 1067,3 & 1342,9 & 4,2 & 5,3 & 2,1 \\
\hline Citrumelo F80-8 & 109,7 & 104,6 & 42,0 & 166,2 & 58,4 & 77,4 & 223,1 & 126,8 & 102,8 & 1011,0 & 1264,4 & 3,7 & 5,8 & 1,8 \\
\hline Citrumelo F80-3 & 43,3 & 53,0 & 25,6 & 90,5 & 53,8 & 27,5 & 126,8 & 81,7 & 49,5 & 551,7 & 708,7 & 2,7 & 4,3 & 2,5 \\
\hline Citrumelo F81-18 & 56,6 & 71,0 & 87,0 & 85,3 & 99,8 & 47,5 & 95,1 & 61,1 & 92,0 & 695,3 & 887,8 & 2,6 & 4 & 3,5 \\
\hline Média & 81,3 & 86,5 & 66,8 & 121,4 & 81,2 & 54,3 & 187,4 & 91,3 & 93,7 & 863,8 & 1102,7 & 3,5 & 4,6 & 2,4 \\
\hline
\end{tabular}

* Em outubro de 2019.

** Eficiência produtiva média do biênio 2018 e 2019.

*** Produção total de frutos das colheitas de 2006 a 2010.

Fonte: Autores.

No quinquênio 2011-2015, (Tabela 2), os citrumelos Swingle (619,6 $\left.\mathrm{kg} \cdot \mathrm{pl}^{-1}\right), \mathrm{W}-2\left(591,3 \mathrm{~kg} \cdot \mathrm{pl}^{-1}\right), \mathrm{F} 80-8\left(480,9 \mathrm{~kg} \cdot \mathrm{pl}^{-1}\right) \mathrm{e}$ F80-7 (466,9 $\left.\mathrm{kg} \mathrm{pl}^{-1}\right)$ novamente induziram as maiores produções à laranjeira Valência. Apesar dos citrumelos F80-8 e F80-7 ocuparem o terceiro e quarto lugares no total do quinquênio, suas posições relativas apresentaram variações: o F80-8 ocupou a primeira posição em 2014, mas em 2013 e 2015 posicionou-se na sétima e oitava colocações enquanto que o F80-7 foi o quarto porta-enxerto em 2013, o nono em 2014 e o sexto em 2015.

Os citrumelos F80-6 (415,5 $\left.\mathrm{kg} \cdot \mathrm{pl}^{-1}\right)$, F81-18 (399,7 $\left.\mathrm{kg}^{-1} \mathrm{pl}^{-1}\right), 4481\left(352,3 \mathrm{~kg} \cdot \mathrm{pl}^{-1}\right), \mathrm{F} 80-5\left(341,7 \mathrm{~kg}^{-1} \mathrm{pl}^{-1}\right)$ e F80-3 $(266,2$ $\left.\mathrm{kg} \cdot \mathrm{pl}^{-1}\right)$, ocuparam as demais colocações, todos com produções inferiores à média geral deste período $\left(437,1 \mathrm{~kg} \cdot \mathrm{pl}^{-1}\right)$.

Também no período seguinte, 2016 - 2019 (Tabela 2), eles proporcionaram produções menores que a média geral do

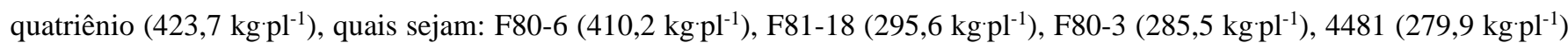

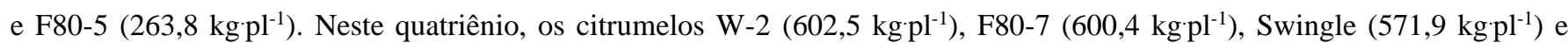
F80-8 $\left(530,1 \mathrm{~kg}^{\mathrm{pl}} \mathrm{l}^{-1}\right)$, novamente foram os mais produtivos.

No total das 14 colheitas (2006 a 2019), os citrumelos W-2 (1.546,4 kg pl-1), Swingle (1.524,2 kg pl-1), F80-7 (1.342,9 $\left.\mathrm{kg} \cdot \mathrm{pl}^{-1}\right)$ e F80-8 $\left(1.264,4 \mathrm{~kg} \mathrm{pl}^{-1}\right)$ favoreceram as maiores produções à laranjeira Valência, todas superiores à média geral do

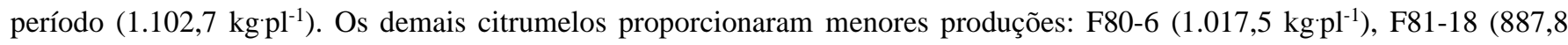
$\left.\mathrm{kg} \cdot \mathrm{pl}^{-1}\right), \mathrm{F} 80-5\left(831,5 \mathrm{~kg} \cdot \mathrm{pl}^{-1}\right), 4481\left(801,2 \mathrm{~kg}^{\mathrm{pl}} \mathrm{l}^{-1}\right)$ e F80-3 $\left(708,7 \mathrm{~kg} \cdot \mathrm{pl}^{-1}\right)$.

As características biométricas das combinações, coletadas em outubro de 2019, quando as plantas tinham 16 anos de idade, revelam que o W-2 desenvolveu as maiores laranjeiras com volume e diâmetro da copa $\left(62,9 \mathrm{~m}^{3}\right.$ e 5,3 m), altura da planta $(4,2 \mathrm{~m})$ e EP $\left(1,7 \mathrm{~kg} \mathrm{~m}^{-3}\right)$ enquanto que o Swingle proporcionou plantas com volume (45,8 $\left.\mathrm{m}^{3}\right)$, diâmetro (4,6 m), altura $(4,1 \mathrm{~m})$ e EP $\left(2,6 \mathrm{~kg} \mathrm{~m}^{-3}\right)$. Já as plantas sobre o citrumelo F80-8 exibiam volume e diâmetro da copa $\left(65,5 \mathrm{~m}^{3} ; 5,8 \mathrm{~m}\right)$, altura $(3,7 \mathrm{~m})$ e EP $\left(1,8 \mathrm{~kg} \mathrm{~m}^{-3}\right)$ e as sobre F80-7 volume $\left(62,6 \mathrm{~m}^{3}\right)$ e diâmetro da copa $(5,3 \mathrm{~m})$, altura $(4,2 \mathrm{~m})$ e EP $\left(2,1 \mathrm{~kg} \cdot \mathrm{m}^{-3}\right)$. Com exceção do F80-6, que apresentou volume de copa $\left(46,2 \mathrm{~m}^{3}\right)$, diâmetro $(4,9 \mathrm{~m})$, altura $(3,6 \mathrm{~m})$ e EP $\left(2,1 \mathrm{~kg} \mathrm{~m}^{-3}\right)$, os demais 
porta-enxertos F80-3, 4481, F81-18 e F80-5 apresentaram volume de copa entre 26,6 e 21,5 m³ , diâmetro entre 4,3 e 3,7 m, altura entre 3,3 e 2,6 m e EP entre 3,5 e 2,6 $\mathrm{kg} \cdot \mathrm{m}^{-3}$.

No primeiro quinquênio, os citrumelos mais produtivos foram W-2, Swingle, F80-7 e F80-8, sendo que W-2 e F80-7 apresentaram os maiores índices de precocidade de produção. Os citrumelos não induziram alternância de produção e não diferiram quanto à eficiência produtiva. Os citrumelos W-2, Swingle e F80-7 proporcionaram as plantas mais altas e com maior diâmetro de copa. No segundo e terceiro períodos, e no total das 14 colheitas, novamente os citrumelos W-2, Swingle e F80-7 proporcionaram as maiores produções e não motivaram alternância de produção. Ao final do experimento, os portaenxertos F80-5, F80-3 e F81-18 induziram plantas com alturas menores que 3,0 m. Apesar de não apresentarem as maiores produções de frutos por planta, eles apresentaram as maiores eficiências produtivas, sendo que o citrumelo F80-5 também apresentou precocidade de produção e menor diâmetro da copa, o que possibilita maior adensamento de plantio.

Trabalhos anteriores envolvendo os citrumelos W-2 e Swingle e a laranjeira Valência, nas condições edafoclimáticas de Cordeirópolis-SP, mostraram que ambos deram origem a plantas igualmente produtivas e de mesmas dimensões (Pompeu Junior \& Blumer, 2011a). Outra característica favorável ao W-2 é a sua compatibilidade com a laranjeira Pera (Pompeu Junior \& Blumer, 2011b). Nas condições deste experimento, o citrumelo W-2 surge como mais uma alternativa ao citrumelo Swingle para regiões endêmicas para a MSC. Ele se assemelhou ao citrumelo Swingle na produção e dimensões das plantas e ambos não mostraram sintomas de incompatibilidade com a laranjeira Valência.

\section{Conclusões}

Decorridos 16 anos do plantio do experimento nenhum dos citrumelos revelou intolerância a morte súbita dos citros ou incompatibilidade (pontuações de goma na linha de enxertia) com a laranjeira Valencia.

No total das 14 colheitas, os citrumelos W-2, Swingle e F80-7 proporcionaram as maiores produções de frutos e não ocasionaram alternância de produção.

O citrumelo F80-5 proporcionou plantas com pequena altura e diâmetro de copa, produção precoce e alta eficiência produtiva permitindo maior densidade de plantio.

\section{Agradecimentos}

À Universidade da Flórida e ao Departamento de Agricultura dos EUA pela cessão dos citrumelos, à Sucocitrico Cutrale S/A pela cessão da área e manutenção do experimento e ao Fundo de Defesa da Citricultura pelo apoio financeiro e empréstimo de veículo. Este projeto recebeu apoio dos Projetos CNPq (Universal 476450/2008-0 e Universal 475553/2010-1).

\section{Referências}

Alvares, C. A., Stape, J. L., Sentelhas, P. C., Moraes, G., Leonardo, J., \& Sparovek, G. (2013) Köppen's climate classification map for Brazil. Meteorologische Zeitschrift, 22(6): 711-728.

Barros, J. C. S.M., Graça, J., Celestino, R. C. A. \& Castro, N. G. (1998) Porta-enxertos para laranjeira Folha Murcha. In: XV Congresso Brasileiro de Fruticultura, 1998, Poços de Caldas, MG. Resumos. p. 271.

Bassanezi, R. B., Bergamin Filho, A., Amorim, L., Gimenes-Fernandes, N., Gottwald, T. R. \& Bové, J. M. (2003). Spatial and temporal analyses of citrus sudden death as a tool to generate hypotheses concerning its etiology. Phytopathology, 93: 502-512.

Bassanezi, R. B., Montesino, L. H., Sanches, A. L., Spósito, M. B., Stuchi, E. S. \& Barbosa, J. C. (2007). Effect of citrus sudden death on yield and quality of sweet orange cultivars in Brazil. Plant Disease, 91:1407-1412.

Bassanezi, R. B., Silva Junior, G. J., Feichtenberger, E., Belasque Junior, J., Behlau, F. \& Wulff, N. A. (2016) Doenças dos citros. In: Manual de Fitopatologia: Doenças das plantas cultivadas, v.2, 271-306.

Beretta, M. J. G., Pompeu Junior, J., Derrick, K. E. \& Lee, R. F. (1994) Evaluation of roostocks in Brasil for field resistance to declinio. In: 1992 International Citrus Congress, Acireale. Proceedings. International Society of Citriculture, p. 841-843. 
Blumer, S. \& Pompeu Junior, J. (2010) Laranjeiras Valência enxertadas em seleções e híbridos dos limões Cravo, Volkameriano e Rugoso na presença da morte súbita dos citros. In: XXI Congresso Brasileiro de Fruticultura, Natal, RN, Anais, cd room. Sociedade Brasileira de Fruticultura.

Bridges, G. D., Youtsey, C. O. (1968). Further studies of the bud-union abnormality of rough lemon rootstocks with sweet orange scions. In: 1966 Conference of the International Organization of Citrus Virologists, Catania. Proceedings. International Organization of Citrus Virologists, p. 236-239.

Cantuarias-Avilés, T., Mourão Filho, F. A. A., Stuchi, E. S., Rodrigues da Silva, S., Espinoza-Nuñes, E. (2011). Horticultural performance of Folha Murcha sweet orange onto twelve rootstocks. Scientia Horticulture, Amsterdam,126 (2): 259-265.

Costa, D. P.; Stuchi, E. S.; Girardi, E. A.; Gesteira, A. S.; Coelho Filho, M. A.; Ledo, C. A. S.; Fadel, A. L.; Silva, A.L.V.; Leão, H.C. \& Ramos, Y.C. (2020), Hybrid rootstocks for Valencia sweet orange in rainfed cultivation under tropical savannah climate. J. Agric.Sci. 12, 40-55.

Costa, D.P., Sanches Stuchi, E., Girardi, E. A., Moreira, A. S., da Silva Gesteira, A., Coelho Filho, M. A. \& dos Santos Soares Filho, W. (2021). Less Is More: A Hard Way to Get Potential Dwarfing Hybrid Rootstocks for Valencia Sweet Orange. Agriculture, 11(4), 354.

Embrapa: Centro Nacional de Pesquisa de Solos (1999) Sistema Brasileiro de Classificação de Solos. p.

Fundecitrus (2021). Guia de Reconhecimento dos Citros em Campo. Website Fundecitrus. https://www.fundecitrus.com.br/.

Geógrafos (2018). Cidades de Minas Gerais. Website Geógrafos. https://www.geografos.com.br/cidades-minas-gerais/comendador-gomes.php.

Laranjeira F.F., Pompeu Jr, J., Garcia, J. R., Vieira, M., Harakava, R. \& Beretta, M.J.G. (1998). Screening for tolerance of citrus to Xylella fastidiosa, the causal agent of citrus variegated chlorosis CVC. Fruits, 53 (5): 345- 349.

Maccheroni, W., Alegria, M. C., Greggio, C. C, Piazza, J. P., Kamla, R. F., Zacharias, P.R., Bar-Joseph, M., Kitajima, E. W., Assumpção, L. C., Camarotte, G., Cardozo, J., Casagrande, E. C, Ferrari, F., Franco, S.F., Giachetto, P. F, Girasol, A., Jordão, H., Silva, V. H., Souza, L. C., Aguilar-Vildoso, C. I., Zanca, A. S., Arruda, P., Kitajima, J. P., Reinach, F. C., Ferro, J. A. \& Da Silva, A. C. (2005). Identification and genomic characterization of a new virus (Tymoviridae family) associated with citrus sudden death disease. Journal of Virology, 79: 3028-3037.

Matsumura, E., Coletta-Filho, H., Nouri, S., Falk, B., Nerva, L., Oliveira, T., Dorta, S. \& Machado, M. (2017). Deep sequencing analysis of RNAs from citrus plants grown in a citrus sudden death-affected area reveals diverse known and putative novel viruses. Viruses, 9 (4): 92-109.

Mendel, K. (1956). Rootstock-scion relationships in Shamouti trees on light soils. Katavim, 6: 35-60.

Müller, G. W., Targon, M. L. \& Machado, M. A. (1999). Trinta anos de uso do clone pré-imunizado 'Pera IAC' na citricultura paulista. Laranja, 20 (2): $399-$ 408.

Moreira, S., Grant, T.J., Salibe, A. A. \& Roessing, C. (1965). Tristeza Tolerant Rootstocks -their behavior after twelve years in orchard- In: 1963 Conference of the International Organization of Citrus Virologists. Proceedings. International Organization of Citrus Virologists, 18-24.

Pimentel Gomes, F. (2009). Curso de estatística experimental. (15a ed.), Fealq. 451p.

Pompeu Junior, J. (2005). In: Citros: Porta-enxertos. Campinas; Instituto Agronômico e Fundag. 929 p.

Pompeu Junior, J. \& Blumer, S. (2002). Incompatibilidade da laranjeira Valência enxertada em híbrido de limão Cravo x citrange Carrizo. In: VII Seminário Internacional de Citros: Melhoramento, Anais. Bebedouro, SP. p.126.

Pompeu Junior, J. \& Blumer, S. (2005). Performance de citrumelos F.80 no Estado de São Paulo. Laranja, 26 (1): 77-85.

Pompeu Junior, J. \& Blumer, S. (2008 a). Morte súbita dos citros: suscetibilidade de seleções de limão cravo e uso de inter-enxerto. Revista Brasileira de Fruticultura, 30: 1159-1161.

Pompeu Junior, J. \& Blumer, S. (2008 b). Laranjeiras e seus porta-enxertos nos viveiros de mudas cítricas do Estado de São Paulo em 2004-2007. Laranja, 29 (único): 35-50.

Pompeu Junior, J. \& Blumer, S. (2011a). Citrumelos como porta-enxertos para a laranjeira Valência. Pesquisa Agropecuária Brasileira, 46 (1): $105-107$.

Pompeu Junior, J. \& Blumer, S. (2011b). Novidades sobre porta-enxertos. XXXIII Semana da Citricultura; Centro de Citricultura Sylvio Moreira, Instituto Agronômico do Estado de São Paulo.

Pompeu Junior, J., Blumer, S. \& Resende, M. D. V. (2013) Avaliação genética de seleções e híbridos de limões Cravo, Volkameriano e Rugoso como portaenxertos para laranjeiras Valência na presença da morte súbita dos citros. Revista Brasileira de Fruticultura, 35 (1): 191-198.

Pompeu Junior, J. \& Blumer, S. (2019). Comportamento de porta-enxertos em área afetada pela morte súbita dos citros. Citrus Research \& Technology, 40, e1048 .

Prestes, R. A., Colnago, L. A., Forato, L. A., Carrilho, E., Bassanezi, R. B. \& Wulff, N. A. (2009). Nuclear magnetic resonance characterization of metabolite disorder in orange trees caused by citrus sudden death disease. Molecular Plant Pathology, 10: 51-57.

R. Core Team (2020). R: A language and environment for statistical computing. R. Foundation for Statistical Computing. URL https://www.R-project.org/.

Rodriguez, O., Rossetti, V., Muller, G. W., Moreira, C. S., Prates, H. S., De Negri, J. D. \& Greve, A. (1979). Declínio de plantas cítricas em São Paulo. In: V Congresso Brasileiro de Fruticultura, Pelotas. Anais. 927-932. 
Research, Society and Development, v. 11, n. 1, e25411124527, 2022

(CC BY 4.0) | ISSN 2525-3409 | DOI: http://dx.doi.org/10.33448/rsd-v11i1.24527

Roman, M., P., Cambra, M., Juarez, J., Moreno, P., Duran-Vila, N., Tanaka, F.A. O., Alves, E., Kitajima, E. W., Yamamoto, P.T., Bassanezi, R. B., Teixeira, D. C., Jesus Junior, W. C., Ayres, A. J., Gimenes-Fernandes, N., Rabenstein, F., Girotto, L. F. \& Bové J. F. (2004) Sudden death of citrus in Brazil: a grafttransmissible bud union disease. Plant Disease, 88: 453-467.

Stenzel, N. M. C., Neves, C. S. V. J., Scholz, M. B. S., \& Gomes, J. C. (2005) Comportamento da laranjeira Folha Murcha em sete porta-enxertos no noroeste do Paraná. Revista Brasileira de Fruticultura, 27(3): 408-411.

Tukey, J. W. (1949). Comparing individual means in the analysis of variance. Biometrics, 5(2): 99-114.

Yamamoto, P. T., Bassanezi, R. B, Wulff, N. A., Santos, M. A., Sanches, A. L., Toloy R. S., Gimenes-Fernandes, N., Ayres, A. J., Jesus Junior, W. C., Nagata, T., Tanaka, F. A. O., Kitajima, E. W. \& Bové, J. M (2011) Citrus sudden death is transmitted by graft-inoculation and natural transmission is prevented by individual insect-proof cages. Plant Disease, 95: 104-112. 